\title{
On winds from giants in binary systems
}

\author{
A. Frankowski and R. Tylenda \\ Nicolaus Copernicus Astronomical Center, Polish Academy of Sciences, Rabiańska 8, 87-100 Toruń, Poland
}

Received 3 July 2000 / Accepted 4 December 2000

\begin{abstract}
We explore the influence of a companion on the wind from a giant in a binary system. We have developed a simple method for estimating the enhancement and intrinsic non-sphericity of the outflow in this case. Assuming the Roche model (modified to account for the reduced effective gravity of the giant), local stellar parameters $\left(T_{\text {eff }}, g\right)$ are calculated. This, according to our approach, allows to derive the local mass loss rate. The influence of varying the model parameters on the results is studied. A relation between the ratio of the stellar radius to the Roche lobe radius and the overall wind enhancement is obtained both numerically and in an analytical approximation. A similar relation is derived for the equatorial-to-polar wind intensity contrast.
\end{abstract}

Key words. binaries: general - stars: mass-loss - stars: late-type - stars: AGB and post-AGB

\section{Introduction}

Mass loss is known to be a key factor for stellar evolution in late stages. Matter escapes easily because of high luminosities and low surface gravities of evolved, cool giants. On the Red Giant Branch (RGB) and especially on the Asymptotic Giant Branch (AGB) the mass loss rate can be larger, sometimes by orders of magnitude, than the nuclear burning rate. Therefore it is mass loss which determines the AGB lifetime and which plays the key role in the stellar initial-final mass relation. Thanks to the stellar winds intermediate mass stars avoid supernova explosions. Instead, due to the copious mass loss terminating the AGB they give origin to planetary nebulae with their amazing shapes.

The stellar winds also affect the evolution of the interstellar medium by influencing its dynamics and spreading elements created in the stellar interiors. It is also believed that interstellar dust has its origin in cool atmospheres and dense winds blowing from the RGB and AGB stars.

The mass loss process becomes even more important, more interesting and more complex when it takes place in a binary system. The most dramatic phenomena occur when an RGB or AGB star happens to fill its Roche lobe. In this case, a dynamically unstable mass transfer to the companion takes place. This leads to the formation of a common envelope and a very rapid evolution of the entire system.

Send offprint requests to: A. Frankowski, e-mail: frankowski@ncac.torun.pl
However, even far from the Roche lobe filling the extended atmosphere of an RGB or AGB star can be significantly disturbed by binarity. The gravitational attraction from the companion and the centrifugal force due to the rotation of the system are expected to increase the mass loss rate. Mass loss from the star also means mass and angular momentum loss from the system. Certain mass and angular momentum accretion on the companion is also possible. The binary parameters are therefore expected to evolve accordingly which, in turn, will affect the stellar mass loss. Thus the evolution of the RGB and AGB stars in binary systems deserves thorough studies as it can be significantly different from that of the single stars.

In this paper we try to investigate effects of binarity on the mass loss rate and the wind structure from cool, evolved stars by analysing intrinsic conditions under which the mass loss process occurs. Generally speaking, mass loss is driven by physical conditions in the stellar atmosphere. Physics involved in mass loss from cool, extended atmospheres is very complex and difficult for theoretical modelling although a significant progress has recently been made in its understanding (e.g. Bowen \& Willson 1991; Arndt et al. 1997). In the classical theory of (plane-parallel) stellar atmospheres the physical structure of the atmosphere, for a given chemical compositon, is determined by two parameters: effective temperature, $T_{\text {eff }}$, and gravity, $g$. In the case of the RGB and AGB stars the situation is certainly more complex. Geometrical extension of the atmosphere and phenomena generated in the stellar interior, e.g. pulsations and convection, influence 
the structure of the atmosphere. The ratio of the photospheric scale height, $H_{\mathrm{p}}\left(\sim T_{\text {eff }} / g\right)$, to the stellar radius, $R$, can be considered as a measure of the geometrical extension. If we define $h_{\mathrm{p}}$ as $H_{\mathrm{p}} / R$ then it seems reasonable to adopt, at least to a first approximation, that $T_{\text {eff }}, g$ and $h_{\mathrm{p}}$ are principal parameters of the RGB and AGB atmospheres and their winds. This notion finds its support in results of modelling winds from the red giants. Bowen \& Willson (1991) have found that the mass loss rate from the AGB stars depends first of all on the density scale height in the atmosphere. Arndt et al. (1997) obtained formulae in which the AGB wind characteristics are functions of the effective temperature, stellar mass, and luminosity. In the famous wind formula for red giants of Reimers (1975), the mass loss rate depends on the stellar mass, radius and luminosity. However, the formulae of Arndt et al. and Reimers can be reduced to dependencies on local atmospheric parameters, i.e. $T_{\text {eff }}, g$ and $h_{\mathrm{p}}$ (see next section). This becomes important in the case of a binary system, where the mass losing star can be noticeably distorted by tidal forces. Differences in the local conditions over the stellar surface may then lead to different local intensities of the outflow. Thus the wind will show an intrinsic directivity and may be globally enhanced.

Next section presents details of our approach. Results of numerical modelling of the wind structure are given and discussed in Sect. 3. An analytical approximation, which helps to understand and interpret the results, is developed in Sect. 4. General discussion and summary are given in Sect. 5. Preliminary results of this study have been presented in Frankowski \& Tylenda (2000).

\section{The numerical model}

Principal assumptions made in our model are the following: 1 . the binary orbit is circular, the giant corotates with the orbital motion, and the gravitational fields of both components can be treated as originating from point masses - hence the Roche model for the effective potential applies; 2 . the stellar surface is defined by the Roche equipotential surface; 3 . the local mass loss rate per unit area, $\dot{m}$, is a function of the local stellar parameters, namely the effective temperature, $T_{\text {eff }}$, gravity, $g$, and the ratio of the photospheric scale height to the stellar radius, $h_{\mathrm{p}}$.

Our formulae for $\dot{m}$ have been derived from relations obtained by other authors, either from theoretical modelling or observations. These relations usually link the global mass loss rate, $\dot{M}$, to the global stellar parameters, i.e. stellar mass, $M$, radius, $R$, and luminosity, $L$. They can be transformed to dependencies on the local atmospheric parameters using standard definitions and relations, i.e. $\dot{m}=\dot{M} / 4 \pi R^{2}, L=4 \pi R^{2} \sigma T_{\mathrm{eff}}^{4}, g=$ $G M / R^{2}$ and $h_{\mathrm{p}}=H_{\mathrm{p}} / R=\left(k / \mu \mathrm{m}_{\mathrm{H}}\right)\left(T_{\text {eff }} / g R\right.$ ) (where $\sigma$ is the Stefan-Boltzmann constant, $G$ is the gravitational constant, $k$ is the Boltzmann constant, $\mu$ is the mean molecular weight, and $m_{\mathrm{H}}$ is the hydrogen atom mass).
In this way the Reimers' (1975) law can be written in the form

$\dot{m} \sim T_{\mathrm{eff}}^{3} h_{\mathrm{p}}$

while Eq. (2) of Arndt et al. (1997) becomes

$\dot{m} \sim h_{\mathrm{p}}^{4.70} T_{\mathrm{eff}}^{-6.84} g^{1.82}$.

Later on in this paper we will not use, however, the mass loss formulae in the form as Eqs. (1) and (2). The reason is that the dependence on $g$ and $T_{\text {eff }}$ is not there easily seen as it is partially hidden in $h_{\mathrm{p}}$. We have found that the discussion and especially the analytical considerations in Sect. 4 become much more clear if $h_{\mathrm{p}}$ is replaced by $T_{\text {eff }} g^{-1} R^{-1}$ in the mass loss formulae. Thus the Reimers' law becomes

$\dot{m} \sim R^{-1} T_{\text {eff }}^{4} g^{-1}$,

while the relation of Arndt et al. is now written as

$\dot{m} \sim R^{-4.70} T_{\mathrm{eff}}^{-2.14} g^{-2.88}$.

The stellar radius which appears in Eqs. (3) and (4) is usually considered as a global stellar parameter. However, for a tidally distorted star the radius can be interpreted as a "local" parameter in a sense that it varies from point to point over the stellar surface.

Now, in general form, we can write:

$\dot{m} \sim R^{a} T_{\text {eff }}^{b} g^{c}$,

where the exponents $a, b$, and $c$ depend on the particular form of the mass loss formula used. An important point here is that gravity, as a counteracting factor to mass loss, appears in the mass loss formulae of this kind with a negative exponent (see Eqs. (3) and (4)). Thus the general pattern is:

$\dot{m} \sim g^{-|c|}$.

In order to obtain the surface distribution of the effective temperature for a tidally distorted star we have applied two approaches. (A) An explicit relation linking $T_{\text {eff }}$ with $g$ of the form:

$T_{\text {eff }} \sim g^{\alpha}$.

This includes the case of a completely uniform temperature distribution $(\alpha=0)$, the von Zeipel's relation (von Zeipel 1924; $\alpha=0.25$ ), and the Lucy's relation (Lucy 1967), developed as a more suitable for convective stars variant of the von Zeipel's relation $(\alpha=0.08)$. (B) An assumption that the luminosity is spread uniformly over the solid angle, and therefore the effective temperature depends only on the radius and the inclination of a given surface element. For a star deep in its Roche lobe this is equivalent to a relation $T_{\text {eff }} \sim g^{0.125}$, but results in a much flatter temperature distribution when the giant starts to fill its Roche lobe. In particular, this allows to avoid the temperature going to zero at the "sub-companion" point (where $g \rightarrow 0$ ), what occurs in the $(\mathrm{A})$ case. 
We have introduced a free parameter, $\gamma$, describing the effectiveness of the Newtonian gravitational force. This has been done to account for effects of pulsation, radiation pressure, and convective motions acting to diminish the gravity on the giant's surface. Thus we assume that the gravitational acceleration from the giant alone is:

$\boldsymbol{g}_{\mathrm{eff}}=-\gamma \frac{G M_{1}}{r^{2}} \frac{\boldsymbol{r}}{r}$.

In the context of early type stars a similar formulation has been proposed e.g. by Schuerman (1972). In our calculations, values of $\gamma$ ranging from 1.0 to 0.1 have been used.

With the assumptions described above, we have calculated sequences of models, representing stars filling an increasing fraction of their Roche lobes for various mass ratios, $q$. The filled fraction of the giant's Roche lobe can be represented by the ratio of the giant's volume radius, $R$, to the Roche lobe volume radius, $R_{\mathrm{RL}}: r_{\mathrm{RL}}=R / R_{\mathrm{RL}}$. (The volume radius is defined as a radius of a sphere of the same volume as the volume of the considered object.) For each point of the stellar surface we have computed radius, gravity and effective temperature (relative to the spherical case). This allowed us to evaluate the local mass loss rate.

Integrating $\dot{m}$ over the whole surface leads to the total mass loss rate, which can be compared with the mass loss rate for a single (spherical) star of the same intrinsic parameters $(L, M, R)$. Further, an intensity contrast between the equatorial and polar mass loss rates can be calculated as a ratio of the local mass loss rate averaged along the stellar equator, $\dot{m}_{\text {eq }}$, to the polar mass loss rate, $\dot{m}_{\text {pol }}$ (the latter is usually close to the minimum value of $\dot{m})$.

\section{Results}

The most important results of our study are presented in Figs. 1-4.

Figures 1a-d show the local mass loss rates represented by grayscale. For each figure black denotes maximum and white - minimum in the mass loss rate. The mass loss rate at the dotted line is equal to that of a single, undistorted (i.e. spherical) star. It marks the border between the polar regions where the mass loss rate is lower, and the "equatorial strip" where it is higher than for the single star. The calculations have been done using the local mass loss rate according to the Arndt et al. prescription (Eq. (4)). The gravity effectiveness factor $\gamma=1.0$ has been adopted. The effective temperature surface distribution follows from the assumption of uniform flux distribution over the solid angle (case (B) described in Sect. 2).

Figures $1 \mathrm{a}-\mathrm{c}$ have been obtained for the mass ratio $q=0.5$ and the ratio of the giant's volume radius to the critical Roche surface volume radius $R / R_{\mathrm{RL}}=0.1,0.5$, 1.0, respectively. The stars are viewed from the orbital plane, with the hemisphere facing the companion being closer to the observer. Figure 1d presents for comparison
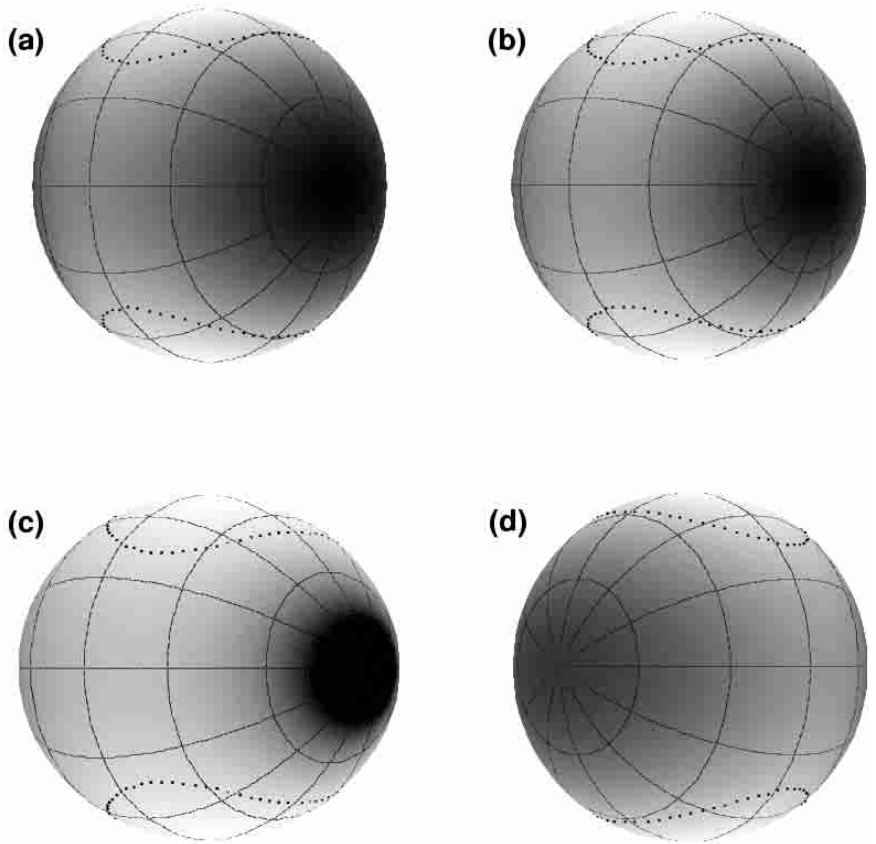

Fig. 1. a-d) The local mass loss rate per unit area across the surface of a giant in a binary system. Dark and light regions have, respectively, high and low mass loss rate per unit area. At the dotted line the local mass loss rate is equal to that of a single star. The mass loss formula of Arndt et al. has been applied. The mass ratio is $q=0.5$. The gravity effectiveness is $\gamma=1.0$. Case (B) of the temperature surface distribution is assumed (see text). a) For the ratio of the stellar radius to the Roche lobe volume radius $r_{\mathrm{RL}}=R / R_{\mathrm{RL}}=0.1$. b) For $r_{\mathrm{RL}}=0.5$. c) For $r_{\mathrm{RL}}=1.0$. d) The same as $\left.\mathbf{b}\right)$, but viewed from the opposite hemisphere

the $q=0.5, R / R_{\mathrm{RL}}=0.5$ case (the same as in Fig. $1 \mathrm{~b}$ ) viewed from the opposite hemisphere.

Figure 2 plots the enhancement of the total mass loss rate caused by the presence of the binary companion, $\Delta \dot{M} / \dot{M}_{\mathrm{s}}$, against the $R / R_{\mathrm{RL}}$ ratio. Note the logarithmic scale on both axes. Points represent the numerical results, lines - the analytical relation derived in Sect. 4, i.e.

$\frac{\Delta \dot{M}}{\dot{M}_{\mathrm{s}}} \sim\left(\frac{R}{R_{\mathrm{RL}}}\right)^{3}$

Thick lines and symbols correspond to $\gamma=1.0$, the thin ones - to $\gamma=0.1$. Figure $2 \mathrm{a}$ has been obtained with the Arndt et al. mass loss formula, while Fig. $2 \mathrm{~b}$ is for the Reimers' law.

Figure 3 presents the wind intensity contrast as a function of the $R / R_{\mathrm{RL}}$ ratio. For clarity of the functional dependence, $\dot{m}_{\text {eq }} / \dot{m}_{\text {pol }}-1$ instead of $\dot{m}_{\text {eq }} / \dot{m}_{\text {pol }}$ is plotted. Again, note the logarithmic scale on both axes. As in Fig. 2, points represent the numerical results, lines - the analytical relation derived in Sect. 4 , i.e.

$\frac{\dot{m}_{\mathrm{eq}}}{\dot{m}_{\mathrm{pol}}}-1 \sim\left(\frac{R}{R_{\mathrm{RL}}}\right)^{3}$. 


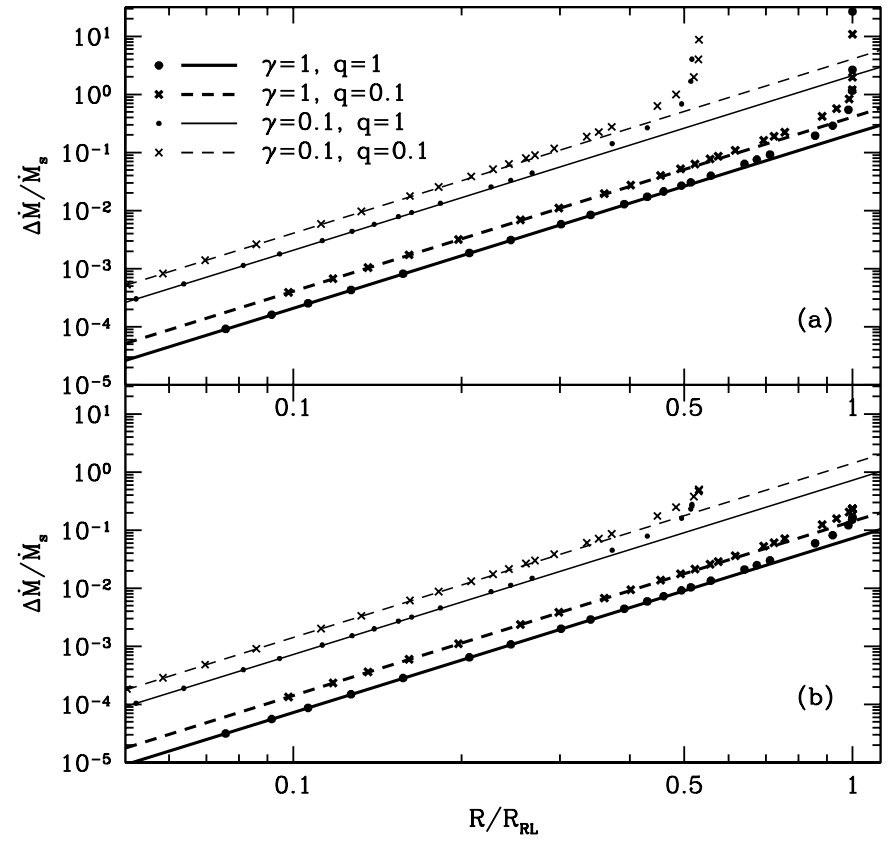

Fig. 2. a and b) The dependence of the total mass loss rate enhancement, $\Delta \dot{M} / \dot{M}_{\mathrm{s}}$, on the $R / R_{\mathrm{RL}}$ ratio: for the mass loss formula of Arndt et al. a) and for the Reimers' law b). The effective temperature surface distribution is uniform. Results for $\gamma=1.0,0.1$ (thick and thin symbols, respectively) and $q=$ 1.0, 0.1 (full and dashed lines, respectively) are shown. Points denote numerical results, lines - analytical relations

The cases of $\gamma=1.0$ and $\gamma=0.1$ are marked in the same way as in Fig. 2. Figure 3a is for the Arndt et al. mass loss formula, Fig. 3b - for the Reimers' law.

Figure 4 shows the influence of the temperature distribution law on the wind intensity contrast. Results of case (A) with $\alpha=0,0.08,0.25$ and of case (B) are displayed for $q=1.0$ and $\gamma=1.0$. As in Figs. 2 and 3, points represent the numerical results, lines - the derived analytical relation. Figure $4 \mathrm{a}$ is for the Arndt et al. mass loss formula, Fig. 4b - for the Reimers' law. In the case of the Reimers' formula, the model with $\alpha=0.25$ is not shown, because it has a reversed intensity contrast (the wind is more intense on the poles than at the equator see Sect. 5).

\section{Analytical approximations}

We have also made analytical considerations in order to obtain approximate expressions for $\Delta \dot{M} / \dot{M}_{\mathrm{s}}$ and for $\dot{m}_{\text {eq }} / \dot{m}_{\text {pol }}$. As usual, analytical formulae help to understand and interpret the numerical results. Our analysis has been done in the limit of small $R / A$ (which is equivalent to small $R / R_{\mathrm{RL}}$ but makes the formulae more clear).

Let us define a corotating spherical coordinate system $(r, \theta, \phi)$, with the origin at the center of mass of the giant, with the $\theta$ angle measured from the axis parallel to the binary rotation axis, and with $\phi$ measured in the orbital plane from the axis pointing to the companion.

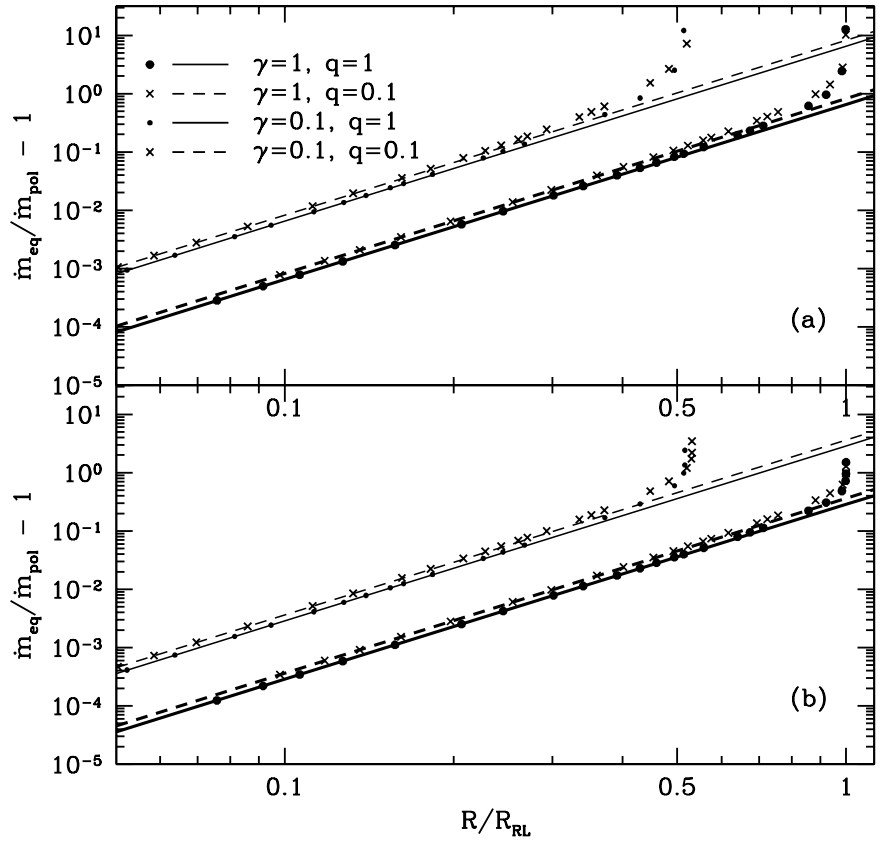

Fig. 3. a and b)The dependence of the equatorial-to-polar wind intensity contrast, $\dot{m}_{\mathrm{eq}} / \dot{m}_{\mathrm{pol}}$, on the $R / R_{\mathrm{RL}}$ ratio: for the mass loss formula of Arndt et al. a) and for the Reimers' law b). The effective temperature surface distribution is uniform. The same notation as in Fig. 2

The stellar surface is then defined as a function $r(\theta, \phi)$. In order to use Eq. (5) for the mass loss rate, we need expressions for $r(\theta, \phi), T_{\text {eff }}(\theta, \phi)$, and $g(\theta, \phi)$ in the considered limit. Actually, we need only formulae for $r$ and $g$, because when $R / A$ is small, $T_{\text {eff }}$ is determined by $g^{\alpha}$ (either in (A) or in (B) case).

For a given point with coordinates $(r, \theta, \phi)$ in the corotating frame, the potential function, $\Phi(r, \theta, \phi)$, is given by:

$\Phi=\gamma \frac{G M_{1}}{r}+\frac{G M_{2}}{r_{2}}+\frac{\omega^{2}}{2} \rho^{2}$,

where $\gamma$ is the giant's gravity effectiveness factor, $M_{1}$ and $M_{2}$ are the masses of the giant and of the companion, respectively, $r_{2}$ is the distance to the companion, $\omega$ is the angular velocity of the binary rotation, and $\rho$ is the distance to the rotation axis, so:

$$
\begin{aligned}
r_{2}^{2} & =A^{2}+r^{2}-2 A r \sin \theta \cos \phi \\
\rho^{2} & =\left(r \sin \theta \cos \phi-\frac{A M_{1}}{M_{1}+M_{2}}\right)^{2}+r^{2} \sin ^{2} \theta \sin ^{2} \phi \\
\omega^{2} & =G\left(M_{1}+M_{2}\right) / A^{3}
\end{aligned}
$$

In a more convenient, dimensionless form (with $\Omega$ being the dimensionless potential) one has:

$\Omega=\gamma \frac{A}{r}+q \frac{A}{r_{2}}+\frac{1+q}{2}\left(\frac{r}{A}\right)^{2} \sin ^{2} \theta-q \frac{r}{A} \sin \theta \cos \phi$,

where $q$ is the mass ratio, $M_{2} / M_{1}$. For a fixed value of $\Omega$, Eq. (11) defines an equipotential surface. Comparing this 


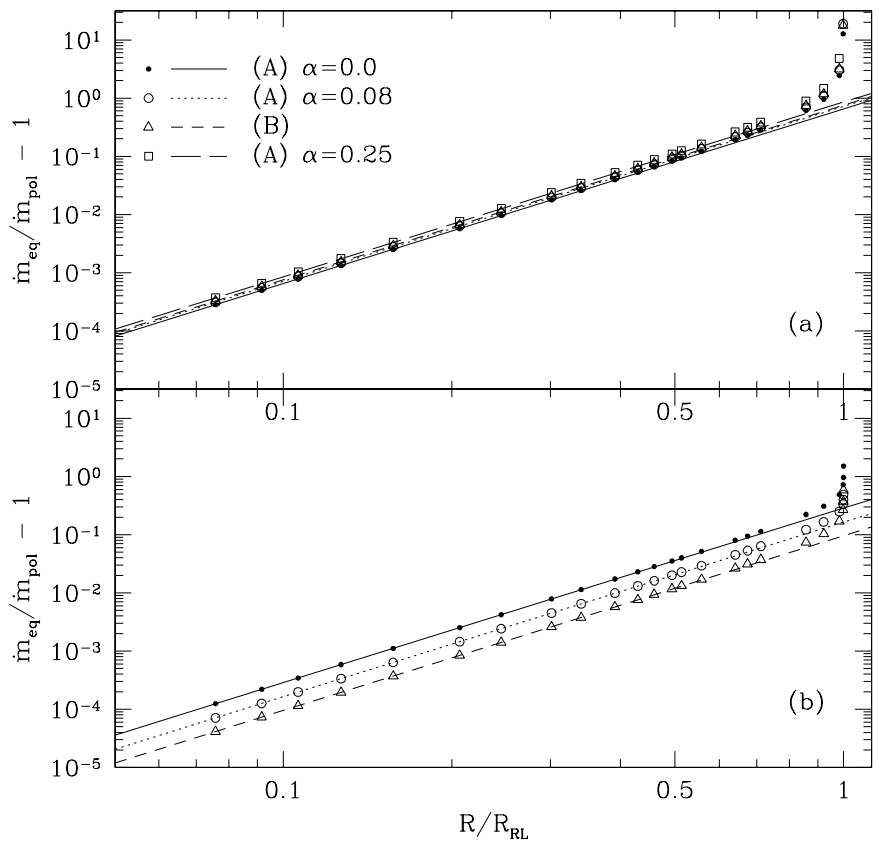

Fig. 4. a and b) The dependence of the equatorial-to-polar wind intensity contrast, $\dot{m}_{\mathrm{eq}} / \dot{m}_{\mathrm{pol}}$, on the $R / R_{\mathrm{RL}}$ ratio for different temperature surface distribution laws: a) the mass loss formula of Arndt et al., b) the Reimers' law. Results for $q=1.0, \gamma=1.0$ are shown. Points denote numerical results, lines - analytical relations

with the equation of surface for a single, spherical star of radius $R$, i.e.

$\Omega_{\mathrm{s}}=\gamma \frac{A}{R}$,

and expanding into a power series in small $r$, one gets (up to terms of order $\left.r^{2}\right)$ :

$\frac{\Omega}{\Omega_{\mathrm{s}}}=\frac{R}{r}+\frac{q}{\gamma} \frac{R}{A}+\frac{1}{2 \gamma} \frac{R r^{2}}{A^{3}} f(\theta, \phi)$,

where we introduced a new symbol, $f(\theta, \phi)$, denoting the angular part of the above expression, i.e.

$f(\theta, \phi)=(1+q) \sin ^{2} \theta+q\left(3 \sin ^{2} \theta \cos ^{2} \phi-1\right)$.

From this point on, the subscript "s" denotes quantities characterizing the single, spherical star of a radius $R$.

The equation of surface of the giant can be written as:

$C=\frac{R}{r}+\frac{R r^{2}}{A^{3}} \frac{1}{2 \gamma} f$

where the constant $C$ also includes the second term in the right hand side of Eq. (13). Now it is easy to obtain the shape of the giant (expanded in $R / A C$ ):

$\frac{r(\theta, \phi)}{R}=C^{-1}\left[1+\frac{1}{2 \gamma} f\left(\frac{R}{A C}\right)^{3}+\ldots\right]$.

The specific value of $C$ for the equipotential surface of the volume radius $R$ can be derived from Eq. (16) and the definition of the volume radius. As a result one gets:

$C=1+\frac{1}{3 \gamma}(1+q)\left(\frac{R}{A}\right)^{3}+\ldots$
This leads to a final expression for the radius, i.e.

$$
\frac{r(\theta, \phi)}{R}=1+\frac{1}{6 \gamma}[3 f-2(1+q)]\left(\frac{R}{A}\right)^{3} .
$$

Note that Eq. (18) approximately describes a triaxial ellipsoid with semi-axes:

$$
\begin{aligned}
& a_{1}=R\left[1+\left(\frac{1}{6 \gamma}\right)(1+7 q)\left(\frac{R}{A}\right)^{3}\right], \\
& a_{2}=R\left[1+\left(\frac{1}{6 \gamma}\right)(1-2 q)\left(\frac{R}{A}\right)^{3}\right], \\
& a_{3}=R\left[1-\left(\frac{1}{6 \gamma}\right)(2+5 q)\left(\frac{R}{A}\right)^{3}\right] .
\end{aligned}
$$

The effective gravity $g(\theta, \phi)$ can be obtained from the gradient of the potential function $\Omega(r, \theta, \phi)$ (Eq. (11)), i.e.

$g(\theta, \phi)=\frac{G M_{1}}{A}|\nabla \Omega|=\frac{G M_{1}}{A}\left(\gamma \frac{A}{r^{2}}-f \frac{r}{A^{2}}+\ldots\right)$.

A comparison with the single star case gives:

$\frac{g(\theta, \phi)}{g_{\mathrm{s}}}=\left(\frac{r}{R}\right)^{-2}\left[1-\frac{1}{\gamma} f\left(\frac{R}{A}\right)^{3}+\ldots\right]$,

or in a convenient form adequate up to terms of order $(R / A)^{3}$ :

$\frac{g(\theta, \phi)}{g_{\mathrm{s}}}=1-\frac{2}{3 \gamma}[3 f-(1+q)]\left(\frac{R}{A}\right)^{3}=\left(\frac{r}{R}\right)^{-4} C^{-2}$.

The proportionality constant in the law linking $T_{\text {eff }}$ with $g$ (Eq. (7)) can be derived from a demand that the stellar luminosity should be the same as in the case of spherical star. This gives:

$\frac{T_{\text {eff }}}{T_{\text {eff }, \mathrm{s}}}=\left(4 \pi R^{2}\right)^{\frac{1}{4}}\left[\int\left(\frac{g}{g_{\mathrm{s}}}\right)^{4 \alpha} \mathrm{d} S\right]^{-\frac{1}{4}}\left(\frac{g}{g_{\mathrm{s}}}\right)^{\alpha}$.

Integrating the flux over the equipotential surface approximated here by a sphere (which is justified for small $R / A$ ) yields:

$\frac{T_{\mathrm{eff}}}{T_{\mathrm{eff}, \mathrm{s}}}=C^{2 \alpha}\left(\frac{g}{g_{\mathrm{s}}}\right)^{\alpha}=\left(\frac{r}{R}\right)^{-4 \alpha}$.

Our mass loss law in the general form of Eq. (5) can be rewritten as:

$\frac{\dot{m}(\theta, \phi)}{\dot{m}_{\mathrm{s}}}=\left(\frac{r}{R}\right)^{a}\left(\frac{T_{\mathrm{eff}}}{T_{\mathrm{eff}, \mathrm{s}}}\right)^{b}\left(\frac{g}{g_{\mathrm{s}}}\right)^{c}$.

Substituting Eqs. (22) and (24) into Eq. (25) one obtains:

$\frac{\dot{m}(\theta, \phi)}{\dot{m}_{\mathrm{s}}}=\left(\frac{r}{R}\right)^{a-4 \alpha b-4 c} C^{-2 c}$.

In the last formula the angular variation of the local mass loss rate is entirely enclosed in the angular variation of $r / R$. $C$ characterizes the given equipotential surface and 
thus is independent of the angles. The factor $C^{-2 c}$ describes the overall wind enhancement, resulting from the modified shape of the potential well.

Now (again making use of the fact that for small $R / A$ we can regard the stellar surface as spherical) an integration of Eq. (26) over the sphere of radius $R$ yields an expression for the global mass loss rate:

$$
\frac{\dot{M}}{\dot{M}_{\mathrm{s}}}=1-\frac{2}{3} \frac{c}{\gamma}(1+q)\left(\frac{R}{A}\right)^{3} \text {. }
$$

The equatorial or polar wind intensity, $\dot{m}_{\text {eq }}$ or $\dot{m}_{\text {pol }}$, can be obtained by putting the respective radius (equatorial, $r_{\text {eq }}$, or polar, $r_{\text {pol }}$ ) into Eq. (26). These radii result from substituting the appropriate values of $\theta$ into Eq. (18) and, in the case of $r_{\text {eq }}$, averaging over the $\phi$ angle. The equatorialto-polar intensity contrast is then:

$\frac{\dot{m}_{\mathrm{eq}}}{\dot{m}_{\mathrm{pol}}}=1+\frac{a-4 \alpha b-4 c}{4 \gamma}(2+5 q)\left(\frac{R}{A}\right)^{3}$.

In order to get formulae dependent on $R / R_{\mathrm{RL}}$, instead of $R / A$, one can simply introduce a new factor $\xi(q)=R_{\mathrm{RL}} / A$ into Eqs. (27) and (28). However, the dependences on $q$ become then apparently more complicated:

$$
\begin{aligned}
& \frac{\dot{M}}{\dot{M}_{\mathrm{s}}}=1-\frac{2}{3} \frac{c}{\gamma}(1+q) \xi(q)^{3}\left(\frac{R}{R_{\mathrm{RL}}}\right)^{3}, \\
& \frac{\dot{m}_{\mathrm{eq}}}{\dot{m}_{\mathrm{pol}}}=1+\frac{a-4 \alpha b-4 c}{4 \gamma}(2+5 q) \xi(q)^{3}\left(\frac{R}{R_{\mathrm{RL}}}\right)^{3} .
\end{aligned}
$$

Equations (29) have been used to derive the analytical lines shown in Figs. 2-4.

\section{Discussion}

We have constructed a model of a tidally enhanced wind from a giant in a binary system. The model has five parameters. Two of them, the mass ratio $q$ and the Roche lobe filling factor $r_{\mathrm{RL}}$, characterize the binary configuration. The other three - i.e. the gravity effectiveness factor $\gamma$, the temperature distribution parameter $\alpha$, and the mass loss law - describe the properties of the mass losing giant. Figures 1-4 and the equations derived in Sect. 4 show the influence of these parameters quantitatively. A more general description and interpretation of the results is given below.

At the beginning we note, that one of the most prominent features of our model (that can be seen from Sect. 4) is that when the Roche lobe filling factor $r_{\mathrm{RL}}$ is small, the departures of all the local mass loss related quantities from their values in the spherical case scale with the third power of $R / A$ (and therefore also with $r_{\mathrm{RL}}^{3}$ ). Other model parameters can be thought of as constituting "scaling factors" for this general dependence. We shall return to this observation in more detail later on.

As can be seen from Eq. (27), the global mass loss rate enhancement depends only on one of three exponents in the mass loss formula (Eq. (5)), namely on the gravity exponent, $c$. The reason for this is evident from Eq. (26), as the $(r / R)^{a-4 \alpha b-4 c}$ term is averaged to 1 when integrating over the stellar surface. The angular dependence of the stellar radius contributes only to the wind intensity contrast. The sign of $c$ decides whether the overall mass loss from the giant in a binary system will be greater (negative $c$ ) or smaller (positive $c$ ) than that of the single giant. As has been noted in Sect. 2 (see comments on Eq. (6)), as gravity counteracts matter ejection, it seems natural to assume $c<0$. Both mass loss formulae considered in this work (Eqs. (3) and (4)) indeed have $c<0$, and so the overall mass loss rate is enhanced compared to the single, non-rotating star case. This is also true for the AGB wind formulae of Blöcker (1995) and for the RGB wind formulae reviewed by Catelan (2000 - with one exception, when a dependence of mass loss on radius only is assumed).

The influence of the mass ratio $q$ is clear when $R / A$ is used as a variable (Eq. (27)) - the higher the mass of the companion, the stronger is the wind enhanced. However, things may look a bit confusing when $r_{\mathrm{RL}}=R / R_{\mathrm{RL}}$ is used (see Eqs. (29) and Fig. 2). The results shown in Fig. 2 seem to be - at first glance - counterintuitive: the lines displaying the wind enhancement for $q=0.1$ lie always above the lines for $q=1.0$. But a greater $q$ means a stronger companion's influence, so why should the global wind enhancement decrease with increasing $q$ ? This is just a result of using $R / R_{\mathrm{RL}}$ as the abscissa. The ratio of the Roche lobe radius to the separation, $\xi(q)=R_{\mathrm{RL}} / A$, decreases with increasing $q$, and this causes the whole expression $(1+q) \xi(q)^{3}$ also to decrease. The increasing $(1+q)$ term does not prevail until $q \gtrsim 5$. Thus - in the range of $q$ considered here - for a given $r_{\mathrm{RL}}$, the overall wind enhancement is in fact greater for a smaller $q$.

The intrinsic structure of the wind in the presence of a companion, as described by our model, can be outlined as follows. For a star well inside its Roche lobe the local mass loss rate per unit area is governed by Eq. (26). This means that maxima and minima of the local mass loss rate coincide with extrema of the stellar radius. These are easy to find, as the stellar surface can be approximated by an triaxial ellipsoid (Eqs. (18) and (19)). The principal axes of the ellipsoid are defined by: the direction to the companion (semi-axis $a_{1}$ ), the direction in the orbital plane that is perpendicular to the latter (semi-axis $a_{2}$ ), and the direction perpendicular to the orbital plane (semi-axis $a_{3}$ ). Equation (19) implies also that $a_{1}>a_{2}>a_{3}$ for any $q>0$.

Magnitudes of the local mass loss rates corresponding to the semi-axes follow this order when the expression $E=a-4 \alpha b-4 c$ is positive, but have a reversed order when it is negative (see Eqs. (26) and (28)). In the first case the global maxima of the mass loss rate occur in the directions to/from the companion, and the global minima occur at the poles. There are also two saddle points, corresponding to the semi-axis $a_{2}$. These are the minima in the $\phi$ coordinate, but maxima in $\theta$. The values of $\dot{m}$ at the saddle points are higher than (or equal to) the value for the spherical case, $\dot{m}_{\mathrm{s}}$, when $2 q(E+2 c) \leq E-4 c$, otherwise they are lower. When comparing $\dot{m}$ at the saddle points 
with the average over the whole stellar surface, $\dot{m}_{\mathrm{av}}$, the analogous condition is $q \leq 0.5$. Thus not always there is a well-defined "equatorial strip" of enhanced mass loss. However, for $E>0$, and provided that $c \leq 0$ (see above), the average equatorial mass loss rate, $\dot{m}_{\mathrm{eq}}$, is enhanced both compared to $\dot{m}_{\mathrm{s}}$, as well as compared to $\dot{m}_{\mathrm{av}}$, independently of the mass ratio $q$. (As a matter of fact, the requirement on $c$ is even less severe, namely $c \leq 0.25 E$, and is needed only when comparing to $\dot{m}_{\mathrm{s}}$.)

In the case of negative $E$ the mass loss is most intense at the poles and weakest in the directions to/from the companion. Also the situation at the saddle points is changed. The condition for $\dot{m} \geq \dot{m}_{\mathrm{s}}$ is still $2 q(E+2 c) \leq$ $E-4 c$, but the one for $\dot{m} \geq \dot{m}_{\mathrm{av}}$ is reversed, i.e. $q \geq 0.5$. Further, if the mass loss from these saddle points is in fact enhanced, then now a "meridional strip" of enhanced mass loss is created. If the opposite is true, there is an equatorial strip of reduced mass loss.

Finally, if $E=0$ the local mass loss rate per unit area is constant over the stellar surface.

For a given mass loss law (a given set of $a, b, c$ ) it is $\alpha$, the temperature distribution parameter, that determines the sign of $E$. In the case of the Reimers' mass loss formula $E$ is positive when $\alpha$ is smaller than 0.1875 . For the Arndt et al. prescription all values of $\alpha$ greater than $\approx-0.80$ result in positive $E$. Since $\alpha<0$ seems unrealistic, all the models with the Arndt et al. mass loss law have an equatorially enhanced mass loss. However, the case of Reimers' formula with $\alpha=0.25$ falls in the region of negative $E$. The wind intensity contrast, $\dot{m}_{\mathrm{eq}} / \dot{m}_{\mathrm{pol}}$, is reversed - the mass loss rate on the poles becomes greater than at the equator. For this reason Fig. $4 \mathrm{~b}$ does not show the case of $\alpha=0.25$. Nevertheless, we may conclude that for the values of $\alpha$ reasonable for stars with deep convective envelopes, our model always gives the equatorial wind enhancement, as expected for cool giants. This holds also for the aforementioned wind formulae of Blöcker (1995) and Catelan (2000).

When the star approaches to fill its Roche lobe, an asymmetry between the sub-companion and offcompanion hemispheres develops. Mass loss towards the companion becomes more intense than in the opposite direction. The asymmetry grows until the gravity at the sub-companion point drops to zero (i.e. when the inner Lagrangian point $L_{1}$ is reached), and the local mass loss rate per unit area at this point becomes infinite.

The above picture remains qualitatively the same independently of the value of $\gamma$. The effect of $\gamma<1$ is essentially to cause the "effective Roche lobe" to shrink. As a result, the giant fills its critical equipotential surface even while it fits comfortably inside its clasically determined Roche lobe. The mass loss enhancement/reduction phenomena described above are more pronounced and the deviations from the ellipsoidal symmetry occur for smaller values of $r_{\mathrm{RL}}=R / R_{\mathrm{RL}}$. The quantitative differences are evident from Eq. (29) and Figs. 2-3. For a given $r_{\mathrm{RL}}$ both the global mass loss enhancement and the equatorial-topolar intensity contrast enhancement scale as $1 / \gamma$.
The use of $\gamma<1$ in our modelling can be justified in different ways. As mentioned in Sect. 2 any kind of motions, like pulsations, waves, turbulence, which are expected to be important in the atmospheres of giants will effectively act as a factor reducing the gravity. In the case of AGB stars there are additional reasons why $\gamma$ significantly smaller than 1 can be expected.

The AGB stars have dusty winds and therefore it seems reasonable to consider, that the intensity of the wind should strongly depend on the conditions in the dust condensation region, which lies well above the photosphere. Replacing in our equations $R$ by the condensation radius, $R_{\text {cond }}$, and assuming, say, $R_{\text {cond }}=2 R$, pushes the maximal possible stellar radius back to $0.5 R_{\mathrm{RL}}$ and increases 8 times the analytical values for the enhancement/reduction of mass loss related quantities. Therefore it is roughly equivalent to setting $\gamma=0.125$. In reality, however, it seems more likely that it is a certain combination of conditions in the stellar atmosphere and in the dust condensation region, that governs the mass loss.

The above considerations are consistent with the results of a study of S stars by Jorissen \& Knapp (1998). These authors do not find any significant mass loss enhancement for the so called "extrinsic S stars" which are believed to be RGB giants in binary systems. However, they find that among the "intrinsic S stars", which are AGB giants, those which are in binaries tend to have the largest mass loss rates.

The assumption made by Bowen (1988) in his models of dusty AGB winds, that the ratio of the acceleration due to radiation pressure on dust to that due to gravity is 0.95 , translates into setting $\gamma=0.05$. The idea of a reduced effective gravity of a mass losing star has also been remarked by Jorissen et al. (1998) in connection with short-period Ba stars.

An interesting result has been obtained by Mürset \& Schmid (1999) from their analysis of symbiotic systems. These authors conclude that the radius of the cool giant in the symbiotic binaries is limited to $R \lesssim 0.5 \ell_{1}$, where $\ell_{1}$ is the distance from the center of the giant to the inner Lagrangian point. This translates into $R \lesssim 0.60-0.70 R_{\mathrm{RL}}$, depending on $q$. Mürset \& Schmid suggest that when the giant exceeds this limit, a dynamical mass transfer ending the symbiotic phenomenon occurs. All the systems involved are s-type symbiotics so they are expected to contain an RGB star. Thus Reimers' formula for the mass loss rate can be applied. The intensity of the outflow from the sub-companion point in our models with the Reimers' wind exhibits a steep rise at $R \approx 0.5 \ell_{1}$ when $\gamma \approx 0.15-$ 0.35 is adopted, depending on $q$.

In theoretical modelling of the evolution of binary systems one has to cope with the fact that the stellar mass loss rate can be affected by the presence of the companion. Tout \& Eggleton (1988) have suggested on a heuristic basis that the wind from a star in a binary system is enhanced by means of tidal forces by a factor of $1+B \times r_{\mathrm{RL}}^{6}$, where $B$ is a free parameter. These authors have proposed $B=10^{4}$. Since then, this prescription has often 
been invoked in various studies of the binary evolution (e.g. Mikołajewska et al. 1989; Tout \& Hall 1991; Soker 1994, 1998; Han et al. 1995; Skopal et al. 1997). However, attempts of establishing a reliable estimate of $B$ have not brought a conclusive result. Han et al. (1995) have preferred $B=500$, whereas in a later work Han (1998) has adopted $B=1000$. Soker et al. (1998) have written the enhancement factor in a more general form, i.e. $1+\mu \times r_{\mathrm{RL}}^{\nu}$ with $\mu$ and $\nu$ being free parameters. From their modelling of off-center planetary nebula nuclei they argue for $\mu=100$ and $\nu=6$.

The numerical and analytical results presented in the present work suggest a different form of the enhancement factor. As has already been mentioned, when the Roche lobe filling factor $r_{\mathrm{RL}}$ is small, the enhancement/reduction of all the local mass loss related quantities scales as $r_{\mathrm{RL}}^{3}$. Also the formula for the global wind enhancement is of the form $1+B^{\prime} \times r_{\mathrm{RL}}^{3}$ (see Eq. (29)). The value of the scaling factor $B^{\prime}$ is again uncertain, but the $1+$ const $\times r_{\mathrm{RL}}^{3}$ pattern is common for all the mass loss related quantities. In fact, it stems from the Roche model geometry and thus should not depend on details of any particular account to the mass loss description. In this paper we have concentrated on the winds from red giants of RGB and AGB, but as we allow for various $\alpha$ exponents in the $T_{\text {eff }}$ vs. $g$ law and for various wind formulae, our approach is not limited to the late type stars only. Our general results should apply to all stars losing mass in the form of wind, irrespectively of their spectral type.

It is worth noting, that the applicability of the above pattern also extends onto single, rotating stars. Although single red giants are generally expected to be slow rotators, and therefore the effects of rotation on the wind should be negligible, recently Heger \& Langer (1998) and García-Segura et al. (1999) conjectured mechanisms that allow single giants at the tip of the AGB to rotate at considerable rates. Our results can be applied also in this case. Indeed, the expression for the global mass loss enhancement (Eq. (27)) with $q=0$ becomes identical to that for a single star rotating with the angular velocity $\omega=\sqrt{G M_{1} / A^{3}}$. For a single star, however, $A$ has a different physical meaning - it is a break-up radius. If one defines the break-up velocity as $\omega_{\mathrm{b}}=\sqrt{G M_{1} / R^{3}}$ then Eq. (27) can be rewritten in a more convenient form for single, rotating stars, i.e.

$$
\frac{\dot{M}}{\dot{M}_{\text {non }- \text { rot }}}=1-\frac{2}{3} \frac{c}{\gamma} \frac{\omega^{2}}{G M_{1}} R^{3}=1-\frac{2}{3} \frac{c}{\gamma}\left(\frac{\omega}{\omega_{\mathrm{b}}}\right)^{2} \text {. }
$$

The same goes for Eq. (28):

$$
\frac{\dot{m}_{\mathrm{eq}}}{\dot{m}_{\mathrm{pol}}}=1+\frac{E}{2 \gamma} \frac{\omega^{2}}{G M_{1}} R^{3}=1+\frac{E}{2 \gamma}\left(\frac{\omega}{\omega_{\mathrm{b}}}\right)^{2} .
$$

To the accuracy of our analytical approximation, this functional dependence of $\dot{M} / \dot{M}_{\text {non-rot }}$ on $\omega / \omega_{\mathrm{b}}$ is the same as that given by Maeder (1999) for radiative winds from rotating early-type single stars.
In this paper we have dealt with the intrinsic structure of the stellar wind, caused by the local differences in physical conditions at the origin of the outflow. Further fate of the ejected matter is beyond the scope of our work. Certainly, the giant's orbital motion and the still present gravitational influence of the companion can change the equatorial-to-polar contrast and complicate the structure of the outflow. Scenarios for such processes have been proposed by Soker $(1994,1997)$ and - along with a detailed numerical modelling - by Mastrodemos \& Morris (1998, 1999). The equatorial compression of the already ejected matter is possible also in winds from single, rotating stars, as shown by Bjorkman \& Cassinelli (1993) and Asida \& Tuchman (1995). The picture can be further enriched by introducing magnetic effects (García-Segura et al. 1999). Our results could be used as inner boundary conditions for considerations of these types.

Acknowledgements. This work has been supported from the grant No. 2.P03D.020.17 of the Polish State Committee for Scientific Research.

\section{References}

Arndt, T. U., Fleischer, A. J., \& Sedlmayr, E. 1997, A\&A, 327, 614

Asida, S. M., \& Tuchman, Y. 1995, ApJ, 455, 286

Bjorkman, J. E., \& Cassinelli, J. P. 1993, ApJ, 409, 429

Blöcker, T. 1995, A\&A, 297, 727

Bowen, G. H. 1988, ApJ, 329, 299

Bowen, G. H., \& Willson, L. A. 1991, ApJ, 375, 53

Catelan, M. 2000, ApJ, 531, 826

Frankowski, A., \& Tylenda, R. 2000, in ASP Conf. Ser., 199, Asymmetrical Planetary Nebulae II: From Origins to Microstructures, ed. J. H. Kastner, N. Soker, \& S. Rappaport, 179

García-Segura, G., Langer, N., Różyczka, M., \& Franco, J. 1999, ApJ, 517, 767

Han, Z. 1998, MNRAS, 296, 1019

Han, Z., Eggleton, P. P., Podsiadlowski, P., \& Tout, Ch. A. 1995, MNRAS, 277, 1443

Heger, A., \& Langer, N. 1998, A\&A, 334, 210

Jorissen, A., \& Knapp, G. R. 1998, A\&AS, 129, 363

Jorissen, A., Van Eck, S., Mayor, M., \& Udry, S. 1998, A\&A, 332,877

Lucy, L. B. 1967, Z. Astrophys., 65, 89

Maeder, A. 1999, A\&A, 347, 185

Mastrodemos, N., \& Morris, M. 1998, ApJ, 497, 303

Mastrodemos, N., \& Morris, M. 1999, ApJ, 523, 357

Mikołajewska, J., Kenyon, S. J., \& Mikołajewski, M. 1989, AJ, 98, 1427

Mürset, U., \& Schmid, H. M. 1999, A\&AS, 137, 473

Reimers, D. 1975, Mem. Soc. Roy. Sci. Liège, 8, 369

Schuerman, D. W. 1972, Ap\&SS, 19, 351

Skopal, A., Vittone, A., Errico, L., et al. 1997, MNRAS, 292, 703

Soker, N. 1994, MNRAS, 270, 774

Soker, N. 1997, ApJS, 112, 487

Soker, N. 1998, ApJ, 496, 833

Soker, N., Rappaport, S., \& Harpaz, A. 1998, ApJ, 496, 842

Tout, Ch. A., \& Eggleton, P. P. 1988, MNRAS, 231, 823

Tout, Ch. A., \& Hall, D. S. 1991, MNRAS, 253, 9

von Zeipel, H. 1924, MNRAS, 84, 702 